Itinéraires Itinéraires

Littérature, textes, cultures

2013-1 | 2013

La fiction aujourd'hui

\title{
Présences paradoxales du romanesque dans la fiction contemporaine
}

Les cas de Jean Echenoz, de Patrick Deville et de Tanguy Viel

\section{Anne Sennhauser}

\section{OpenEdition}

\section{Journals}

Édition électronique

URL : http://journals.openedition.org/itineraires/807

DOI : 10.4000/itineraires.807

ISSN : 2427-920X

Éditeur

Pléiade

\section{Édition imprimée}

Date de publication : 1 octobre 2013

Pagination : 65-79

ISBN : 978-2-343-01791-4

ISSN : 2100-1340

Référence électronique

Anne Sennhauser, «Présences paradoxales du romanesque dans la fiction contemporaine »,

Itinéraires [En ligne], 2013-1 | 2013, mis en ligne le 01 octobre 2013, consulté le 20 avril 2019. URL :

http://journals.openedition.org/itineraires/807 ; DOI : 10.4000/itineraires.807

\section{(9) $\Theta \Theta \Theta$}

Itinéraires est mis à disposition selon les termes de la licence Creative Commons Attribution - Pas d'Utilisation Commerciale - Pas de Modification 4.0 International. 


\title{
Présences paradoxales du romanesque dans la fiction contemporaine
}

\author{
Les cas de Jean Echenoz, \\ de Patrick Deville et de Tanguy Viel
}

\section{Résumé}

Depuis les trente dernières années, la réécriture s'est imposée comme le signe d'une nouvelle approche de l'héritage littéraire. Des œuvres comme celles de Jean Echenoz, Patrick Deville et Tanguy Viel expriment en effet, au-delà de la simple reprise parodique, une fascination pour toute une mythologie de l'aventure et du crime, qui renvoie à une veine romanesque souvent décriée. Chez les trois auteurs étudiés, cette dernière devient vectrice de renouvellements profonds, dans la mesure où elle permet moins de restaurer des modèles désuets que d'en explorer les stéréotypes narratifs et d'interroger le statut de l'imagination. La fiction s'en trouve légitimée d'un point de vue esthétique et ontologique, étant désignée comme un plaisir de lecture aussi bien que comme un art de vivre.

Mots clés : roman français contemporain, Jean Echenoz, Patrick Deville, Tanguy Viel, effet de fiction, imagination

\section{Abstract}

For the last thirty years, rewriting has proven to be a new approach to literary heritage: works such as those by Jean Echenoz, Patrick Deville and Tanguy Viel are more than mere parodies. They show a fascination with the mythology of adventure and crime which refers to romance, inspiration often criticized. Nevertheless, it appears to extensively remodel the writings of these authors, for their stories explore narrative stereotypes and question the status of the realm of fancy rather than restore outdated models. By these means, fiction is legitimized both aesthetically and ontologically, since it is offered both for reading pleasure and as an art of living.

Keywords: contemporary French novel, Jean Echenoz, Patrick Deville, Tanguy Viel, fiction effect, imagination 
Dans un article consacré à la notion polysémique de « romanesque », Jean-Marie Schaeffer prend ses distances vis-à-vis d'une catégorie fréquemment convoquée pour caractériser le renouveau littéraire des années 1980 : plutôt que de « retour "postmoderne" à la fiction », il propose de parler d'une « réactivation de la veine romanesque ${ }^{1}$ », veine qu'il conçoit comme une catégorie privilégiant l'intensité des sentiments, la multiplicité des événements et la prédilection pour 1'extraordinaire, c'est-à-dire caractérisée par l'outrance tant d'un point de vue esthétique que d'un point de vue éthique. Prenant ainsi ses distances avec la notion controversée de postmoderne, qui insiste davantage, par sa morphologie même, sur l'idée de fin que sur celle de renouvellement, il propose d'envisager les pratiques littéraires spécifiques nées dans les années 1980 non pas comme une liquidation de la modernité mais comme un mouvement de réappropriation de la puissance fictionnelle du roman - le romanesque apparaissant comme une forme hyperbolique de fiction. Cette présence du romanesque dans le paysage littéraire contemporain témoigne, lorsqu' on la replace dans le contexte plus vaste de l'évolution du roman, d'une inflexion notable : se penchant sur la naissance du roman moderne, Yves Hersant a en effet pu souligner que ce dernier s'était construit « contre le romanesque ${ }^{2}$ »; le rejet de cette catégorie peut même être considéré, selon Thomas Pavel, comme une caractéristique historique du roman moderne, qui abandonne la vision d'un monde idéalisé pour refuser l'invraisemblance de la pastorale, des romans héroïques ou, plus tardivement, des sous-genres de la littérature d'action ${ }^{3}$. À travers le choix du romanesque, c'est donc toute une tradition vériste qui est mise à distance : la volonté d'authenticité et ses conséquences sur la fiction sont rejetées au profit d'une attitude décomplexée dont l'un des modèles est à chercher du côté de George Perec. L'œuvre de Jean Echenoz fait précisément écho à cette attitude, ce dont témoigne son premier roman, Le Méridien de Greenwich, paru en 1979 et caractérisé par la place paradoxale accordée à un genre qu'il subvertit tout en cherchant à maintenir une « exaltation de l'écriture romanesque ${ }^{4}$ ».

Depuis les années 1980, l'œuvre de cet auteur a évolué - elle est riche aujourd'hui de quinze romans - et a stimulé d'autres pratiques d'écriture chez des écrivains qui reconnaissent volontiers leur dette envers leur aîné des éditions de Minuit. Patrick Deville, dont le premier roman, Cordonbleu, date de 1987, et Tanguy Viel, qui fait son entrée sur la scène littéraire

1. Jean-Marie Schaeffer, "La catégorie du romanesque », dans Michel Murat et Gilles Declerq (dir.), Le Romanesque, Paris, Presses Sorbonne nouvelle, 2004, p. 291-302.

2. Yves Hersant, «Le roman contre le romanesque », L'Atelier du roman, $\mathrm{n}^{\circ} 6$, printemps 1996, p. 145-153.

3. Thomas Pavel, «Les sources romanesques du roman », L'Atelier du roman, $\mathrm{n}^{\circ} 10$, printemps 1997, p. 139-161.

4. Jean Echenoz, «Il se passe quelque chose avec le jazz », Europe, n 820-821, aoûtseptembre 1997, p. 194. 
en 1998 avec Le Black Note, illustrent ainsi de manière singulière une fascination pour des modèles romanesques connus en choisissant eux aussi de répondre positivement à l' « invitation au mensonge ${ }^{5}$ » lancée par la fiction. Alors que les romanciers de " l'ère du soupçon » s'attachaient à stigmatiser la facticité du discours narratif et à dépouiller le récit de ses leurres pour orienter le roman vers l'angoisse du néant, ils ne reculent pas devant le foisonnement narratif et choisissent de se confronter au doute par le biais de l'outrance et des exubérances de l'imagination. Le détour par les sous-genres de la littérature d'action permet chez Jean Echenoz, Patrick Deville et Tanguy Viel de faire du plaisir de la narration un enjeu central et de manifester une prédilection pour une série de topoï romanesques variés - aventures et enquêtes, crimes et courses-poursuites, secrets familiaux...voire pour des situations hautement rocambolesques - visite du purgatoire chez Jean Echenoz, vie conjugale avec un mannequin de verre chez Patrick Deville, déboires d'un assassin déguisé en clown chez Tanguy Viel. Ce goût pour le fantasque et la profusion événementielle renvoie bien à première vue à la catégorie romanesque définie plus haut, mais la réactivation dont parle Jean-Marie Schaeffer est ici rendue problématique dans la mesure où contrairement aux romans qualifiés de romanesques, qui génèrent l'adhésion à une logique de la rêverie ${ }^{6}$, les romans qui nous occupent ne cherchent pas à dissimuler leur invraisemblance, à faire coïncider l'illusion et le réel et à réinstaurer une lecture axiologique du monde. Ils associent davantage la catégorie du romanesque à une réflexion sur la fiction et sur les interférences complexes entre réalité et imaginaire, jusqu'à mettre en doute la possibilité d'une distinction entre le vrai et le faux. Si détour par le romanesque il y a, ce détour semble être mis au profit d'une réévaluation de la fiction non pas en ce qu'elle permet de reproduire mimétiquement le réel mais en ce qu'elle est partie prenante d'un rapport au monde. Le romanesque est ainsi renvoyé à ses ressorts les plus intimes : ceux d'un hédonisme esthétique plutôt que de l'axiologie d'une vision idéalisée du monde.

Il s'agit dès lors d'étudier l'exploitation narrative des motifs romanesques - des thèmes de l'amour exalté, de l'expédition aventureuse, de l'enquête insolite ou encore de la transgression sociale à laquelle renvoient le meurtre, le cambriolage ou l'incendie criminel - pour montrer en quoi elle permet de renouveler l'écriture tout en conceptualisant, au sein d'une diégèse spéculaire, la compétence fictionnelle de l'individu et la place irréductible de l'imaginaire dans l'expérience humaine. Si la narration subversive instaurée par Jean Echenoz, Patrick Deville et Tanguy Viel met à distance les stéréotypes - le traitement des formes romanesques chez ces

5. Gilles Barbedette, L'Invitation au mensonge, Paris, Gallimard, 1999.

6. Cette distinction renvoie à la ligne de partage qui distingue la veine idéaliste de la romance (Richardson, Rousseau) et la veine critique de la novel (Sterne, Diderot), dont Northrop Frye souligne qu'elle oppose participation empathique et distanciation ironique (L'Écriture profane, essai sur la structure du romanesque, [Saulxures], Circé, 1998). 
auteurs a souvent été étudié dans l'optique d'une « esthétique du dégage-

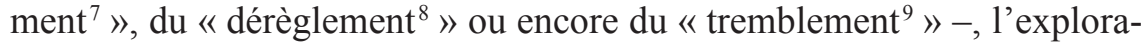
tion thématique du romanesque introduit une réhabilitation à la fois esthétique et ontologique de la fiction. La mise en place d'un cadre spéculaire permet le déploiement d'une dynamique jubilatoire de la déréalisation qui est aussi prospection ontologique.

\section{Un romanesque spéculaire}

Parce qu'ils puisent dans l'imaginaire romanesque une matière foisonnante qui nourrit la narration, les romans dégagent les caractéristiques incarnant selon Alain Schaffner une aspiration au romanesque : « le goût retrouvé de la fiction, le plaisir de l'intrigue et des péripéties, l'amour du personnage excentrique, le lien renoué avec le référent, le jeu avec une tradition littéraire de nouveau pleinement assumée ${ }^{10} \gg$. Ces éléments participent à la mise en valeur d'une facticité revendiquée et le texte désigne en creux ses modèles, ses images, ses figures tutélaires, en se rattachant à tout un univers de signes constitués. Les auteurs n'hésitent pas en effet à adosser leurs récits à un inventaire générique dont ils désignent certains modèles de manière explicite : ceux de Robert-Louis Stevenson ou de Marcel Schwob chez Jean Echenoz et Patrick Deville ou encore celui, cinématographique, d'Alfred Hitchcock désigné en filigrane dans La Tentation des armes à feu de Patrick Deville et dans plusieurs romans de Tanguy Viel. Si Jean Echenoz a souligné à de nombreuses reprises son désir de vagabondage générique et sa stratégie de dérive par rapport aux modèles, Patrick Deville qualifie lui-même ses six premières fictions, parues entre 1987 et 2000 aux éditions de Minuit, de « romans expérimentaux du Xx ${ }^{\mathrm{e}}$ siècle $^{11}$ », c'est-àdire de variations critiques à partir de modèles romanesques, qui jouent sur les effets narratifs des schémas convenus du roman policier, du roman d'aventures ou de la romance. Le romanesque ne va pas sans la conscience du recyclage de motifs scripturaux, souvent déclassés, les auteurs prolongeant les conclusions de l'ère du soupçon pour affirmer qu'il n'y a pas de réalité première en dehors d'un ensemble de récits déjà constitués. C'est en

7. Dominique Viart, «Jean Echenoz et l'esthétique du dégagement», dans Christine Jérusalem et Jean-Bernard Vray (dir.), Jean Echenoz, une tentative modeste de description $d u$ monde, Saint-Étienne, Publications de l'université de Saint-Étienne, 2006, p. 243-254.

8. Henri Scepi, «Patrick Deville : pour une esthétique du dérèglement », Critique, n 523 , décembre 1990, p. 1020-1041.

9. Roger-Michel Allemand, «Tanguy Viel, imaginaires d'un romancier contemporain », @nalyses, [En ligne], http://www.revue-analyses.org/index.php?id=1217 (consulté le 30 juin 2011).

10. Alain Schaffner, «Le romanesque mode d'emploi », dans Marc Dambre et Wolfgang Asholt (dir.), Un retour des normes romanesques dans la littérature française contemporaine, Paris, Presses Sorbonne nouvelle, 2010, p. 51-65.

11. "Vingt mille lieux sur la terre», propos recueillis par Jean-Maurice de Montrémy, Livres Hebdo, nº 758, 12 décembre 2008, p. 74. 
ce sens que Tanguy Viel parle de la littérature comme d'un regroupement hétéroclite de bribes de discours, qu'il dit aller chercher tant du côté de l'imaginaire du crime que du côté de la littérature gothique du XIX siècle ou du côté des modèles cinématographiques ${ }^{12}$. L'isotopie de la fiction forme en outre un métalangage qui se déploie parallèlement à la diégèse et la déréalise en la présentant comme construction esthétique. Au-delà des hypotextes explicitement désignés, le foisonnement des images du roman, la représentation des activités de lecture et d'écriture, thématisent le texte littéraire et renvoient au roman la représentation de sa facticité : le métafictionnel prend ainsi une place centrale dans la stratégie d'écriture, ne serait-ce que par la récurrence de figures de narrateurs-écrivains, que l'on retrouve dans près de la moitié des romans de Patrick Deville, dans Paris-Brest de Tanguy Viel ou que l'on devine à travers les figures d'inventeurs dans les romans de Jean Echenoz.

Face à cette visibilité des ressorts de la fiction, les derniers romans des trois auteurs en question peuvent paraître en retrait : une rupture se fait en effet nettement sentir dans la mesure où l'intrigue foisonnante, la multiplicité des archétypes romanesques identifiables, laisse place à un resserrement de l'action autour d'un personnage singulier ancré dans une réalité souvent factuelle. La série echenozienne de ce que la critique a désigné sous le nom de « biofictions »-Ravel (2006), Courir (2008), Des éclairs (2010) -, les enquêtes sur la vie de grands explorateurs ou de figures littéraires chez Patrick Deville - Pura vida, vie et mort de William Walker (2004), La Tentation des armes à feu (2006), Equatoria (2009), Kampuchéa (2011) - et le roman familial chez Tanguy Viel - Paris-Brest (2009) - renoncent en effet à la complexité d'une intrigue extensible pour faire de fragments de vie le canevas de la fiction. Mais l'ancrage revendiqué dans le vécu - personnages réels, résonances autobiographiques - est cependant loin d'effacer toute charge romanesque et toute volonté de jouer de la facticité du texte, du déploiement de l'invention. Sans obéir à la logique des romans historiques, biographiques ou autobiographiques, qui sont convoqués pour être mis à distance, et en poursuivant leur entreprise de subversion, les auteurs brouillent la référentialité de leurs textes et passent le référent factuel au crible de la déconstruction ou de l'exagération. Une logique fictionnalisante prévaut ainsi dans Paris-Brest, où le « roman familial » renvoie à l'extrapolation romanesque qui le fonde : « Dans mon livre, oui, je me suis dit que ce serait mieux comme ça, que Kermeur ait fait de la prison à cause du cambriolage. Ce serait plus romanesque ${ }^{13}$. »

Dans sa quête historique, le narrateur de Pura vida a par ailleurs bien conscience de ne pouvoir se confronter qu'à des figures distantes et nimbées de légendes romanesques. Il désigne Simon Bolivar de la sorte :

12. Roger-Michel Allemand, op. cit.

13. Tanguy Viel, Paris-Brest, Paris, Minuit, 2008, p. 176. 
Celui qu'il est difficile aujourd'hui d'imaginer sans penser au personnage que fit de lui le romancier colombien Gabriel García Márquez, tout comme il est impossible d'imaginer les derniers jours d'Alcibiade, ou d'Alexandre, sans retrouver les phrases de Plutarque ${ }^{14}$.

L'abondance des noms réels dans cette périphrase descriptive, loin de sous-estimer le processus déformant de l'imagination, en fait la pierre de touche de la mémoire.

Si les débats théoriques sur ce qui fait le propre de la fiction sont loin d'être clos, il n'en reste pas moins que les romans qui nous occupent cherchent à générer un effet de fiction, proclamant leur caractère subjectif dans l'acte de langage qui les constitue. Le concept de « ficsemblable ${ }^{15}$ », défini par Sophie Rabau à l'issue d'une réflexion sur l'effet de fiction, peut nous aider à analyser cette volonté de mettre mal les garants de l'authenticité fictionnelle. Il permet de désigner le pacte de lecture établi par certains discours sérieux qui se présentent comme fictifs : « symétriquement inverse au vraisemblable ${ }^{16} »$, le ficsemblable équivaut ainsi à l'effet de fiction développé par un énoncé factuel, et semble pouvoir être opérant pour comprendre le fonctionnement de tout type de discours cherchant à briser l'illusion référentielle et à souligner son manque d'authenticité. Les garants de la fiction - la figure du narrateur et la vraisemblance de son discours sont précisément mis à mal, les effets de fiction envahissant à divers niveaux une parole qui se dénonce sans cesse comme « feintise ludique ${ }^{17}$ », selon l'expression de Jean-Marie Schaeffer. En se faisant réflexif et en exhibant son processus de transformation, le récit appelle à une conscience pleine de sa facticité en tentant de restaurer une dynamique romanesque qui passe le référent au crible de l'imagination, par-delà la frontière entre le factuel et l'inventé.

\section{Des esthétiques de la déréalisation fictive}

La logique de la fiction prévaut ainsi à travers la mise à mal de la vraisemblance qui, comme le soulignent France Fortier et Andrée Mercier dans leur analyse de l'autorité fictionnelle ${ }^{18}$, fonde traditionnellement la validité du discours. Les matériaux de la fiction, quelle que soit leur nature, sont soumis à une dynamique inventive apte à produire un romanesque qui n'obéit pas à une logique mimétique calquée sur un principe de

14. Patrick Deville, Pura vida, vie et mort de William Walker, Paris, Le Seuil, 2004, p. 37.

15. Sophie Rabau, « Du ficsemblable : brève proposition », Atelier de théorie littéraire, [En ligne], http://www.fabula.org/atelier.php?Ficsemblable (consulté le 30 juin 2011).

16. Ibid.

17. Jean-Marie Schaeffer, Pourquoi la fiction?, Paris, Le Seuil, 1999.

18. France Fortier et Andrée Mercier, «Ces romans qui racontent. Formes et enjeux de l'autorité narrative contemporaine », dans René Audet (dir.), Enjeux du roman contemporain, Québec, Nota Bene, 2009, p. 255-275. 
vraisemblance mais qui déploie plutôt les possibles du récit. La puissance d'invention se veut ici logique d'écriture : l'incongru, l'inattendu, le fantasque en sont les signes les plus visibles, qui renvoient à la dimension fabulée du récit. La référence au récit d'aventures, omniprésente chez les trois auteurs, est ici éclairante puisqu'elle permet de comprendre en quoi, au-delà des motifs les plus éculés du voyage exotique ou de l'expédition hérö̈que auxquels les auteurs ne réduisent pas la puissance romanesque, les récits cherchent à reconduire une dynamique de l'imprévu qui repose sur le surgissement de l'insolite et le déploiement des possibles. Si le récit d'aventures est souvent associé par la critique à une forme hyperbolique de la fiction ${ }^{19}$, c'est bien parce que ce dernier multiplie les surprises et repose, selon les mots de Jacques Rivière, sur « ce qui s'ajoute, ce qui arrive par-dessus le marché, ce qu'on n'attendait pas, ce dont on aurait pu se passer ${ }^{20} »$. La mise à mal des balises rationnelles semble justement permettre la surprise. Elle passe par une contestation réjouie de la vraisemblance empirique, diégétique et énonciative, les auteurs ne reculant pas devant la possibilité de contradictions ou d'apories, au nom même de la nature fictionnelle de leurs textes.

Si la poétique classique préfère ce qui est « impossible mais vraisemblable » à ce qui est « possible mais non persuasif ${ }^{21}$ », les trois auteurs, de manière différente, refusent de soumettre le récit à un principe de rationalité. La logique narrative est subvertie par des procédés de distorsion et de parcellisation, non pas qu'il s'agisse de laisser au lecteur le soin d'en reconstruire le sens sous-jacent, mais parce qu'elle est essentiellement dépourvue de motivation vraisemblable. Le déroulement de l'action porte les traces de ce processus : les voyages aporétiques chez Jean Echenoz, les rencontres sans retombées chez Patrick Deville ou les machinations inefficaces chez Tanguy Viel offrent autant d'exemples d'une action qui n'est pas téléologique, mais qui tourne à vide et qui apparaît comme un leurre. Le régime d'invraisemblance diégétique prédomine ainsi dans la mesure où l'action est rendue dérisoire du fait d'une narration fantasque ou erratique, dont le mouvement prend bien souvent la diégèse à rebours en développant une logique seconde qui se substitue aux événements annoncés et attendus.

Les premiers romans de Patrick Deville offrent de nombreux exemples d'une narration fantasque, à l'image de la désinvolture revendiquée par Jean Echenoz : l'extrapolation romanesque fonde ainsi les intrigues du $\mathrm{Feu}$ d'artifice où il est question d'un tour d'Europe onirique et incongru, de La Femme parfaite, récit de la vie fantasmée d'un agent diplomatique, ou encore de Ces deux-là, huis clos insulaire de trois couples à la dérive. Dans

19. Daniel Couégnas et Alain-Michel Boyer (dir.), Poétiques du roman d'aventures, Nantes, Cécile Defaut, 2004.

20. Jacques Rivière, «Le roman d'aventure (fin) », La Nouvelle Revue française, $\mathrm{n}^{\circ} 55$, juillet 1913, p. 65.

21. Aristote, La Poétique, Paris, Le Seuil, 1980, p. 127. 
ces romans, les nombreux déplacements des personnages ne semblent obéir à aucune logique vraisemblable, suggérant ainsi que le peu de sens que les personnages pensent pouvoir donner à leur existence - une rencontre amoureuse, un acte de bravoure - n'est en réalité que le fruit du hasard. Le roman Ces deux-là cherche ainsi à répondre à l'interrogation simple générée par la vision de deux couples : « Pourquoi ces deux-là, à table? Et ces deux autres, là-bas, et pas l'inverse ${ }^{22}$ ? »Cette question posée en amont du texte débouche sur le déploiement complexe de six trajectoires qui se confondent ou se dissocient au gré des circonstances, dans un ballet de lignes qui ne convoque pas une logique rationnelle mais rappelle l'image du «feu d'artifice » évoquée dans le roman éponyme. La description de ces fusées qui « filaient vers la lune en mouvements sinueux ou paraboliques » et « rejoignaient la surface de l'eau avec un grésillement d'escarbilles et d'escarboucles rouges survolées de fumerolles ${ }^{23}$ » traduit très bien les avancées aléatoires et fulgurantes de personnages attirés par la vitesse et les points culminants, la mobilité désorientée se faisant caractéristique de leur présence au monde. Les lignes sinueuses du feu d'artifice servent de métaphore à toute une logique existentielle ainsi qu'à une esthétique narrative privilégiant la progression heurtée, l'attention portée aux surfaces colorées et aux formes mouvantes. Les romans de Patrick Deville montrent des protagonistes qui progressivement découvrent «- amusant, d'en finir aussi tard avec l'aristotélisme - que le mouvement d'un mobile dans l'espace est facile et gratuit ${ }^{24} \gg$, et qui voient coexister en eux une sensation pleine de l'existence et la conscience de leur précarité.

Les narrations erratiques que l'on trouve chez Tanguy Viel relèvent d'une logique autre, qui participent cependant au même effet de déréalisation. Les narrateurs marqués par l'amnésie ou par l'obsession s'attachent en effet à revenir sans cesse sur leurs récits non pas en soulignant leur vacuité mais en exhibant la difficulté à en fixer leur sens. L'hypertrophie de l'imagination est ainsi rendue sensible dans un flot narratif que les obsessions et les revirements viennent déstructurer. La contradiction occupe une place centrale dans des romans où les narrateurs souvent présentés comme herméneutes - ils cherchent par la réécriture à s'approprier le sens d'une histoire, celle d'un film dans Cinéma ou celle de leur propre vie dans Paris-Brestmultiplient les affirmations contraires. C'est le cas par exemple du narrateur du Black Note, dont le discours constitue une éparnothose qui n'a de cesse de transformer les affirmations qui visent à établir le déroulement d'un crime dans lequel le narrateur - on ne l'apprend que progressivement - est directement impliqué. Parce que le narrateur ne maîtrise pas son discours et qu'il est hanté par ce qu'il nomme « ses fantômes », la logique irrationnelle est poussée à son comble pour amener à une réinterprétation constante de

22. Jean-Didier Wagneur, « Deville dans l'œuf du cyclone », Libération, 26 octobre 2000.

23. Patrick Deville, Le Feu d'artifice, op. cit., p. 155.

24. Ibid., p. 50. 
l'action et frapper l'ensemble du récit d'irréalité, comme cela est également le cas dans le roman Insoupçonnable. Le discours exhibe sa propre insuffisance, déstabilise l'autorité du narrateur et le statut d'un récit qui peine à générer un sens - tant sur le plan logique que sur le plan existentiel.

Moins que dans le fil de l'intrigue, progressivement évidé, ce sont dans les contrepoints inattendus, les ellipses et les extrapolations, que vient se loger la dynamique romanesque : elle joue avec les codes de la vraisemblance d'une intrigue qui se veut dès lors propice au surgissement de l'insolite, qui multiplie les coïncidences ou les détours retors. Les détails d'une scène, objets ou gestes anodins, deviennent le support d'un déploiement des possibles : chez Jean Echenoz, la vue d'un ticket de métro suffit à déclencher l'énumération de ses usages possibles et à sonder ce faisant des pratiques et des maux d'époque; chez Patrick Deville, la présence d'une simple pierre ou d'un cours d'eau génère des sauts spatiaux et temporels qui retracent une généalogie imaginaire, tandis que chez Tanguy Viel, un objet quelconque peut se faire support d'une plongée dans l'intériorité bousculée du narrateur. Chez les trois auteurs, le détail inattendu fait dévier la narration vers des possibles lointains ou enfouis, qui distordent le système causal et chronologique : la place prépondérante donnée à cet imprévu permet de faire surgir un élément hétérogène qui dérange les cadres déterminés de la narration et agit comme révélateur. La dynamique romanesque est ainsi fondée sur un jeu avec les codes de la vraisemblance et une exhibition de la toute-puissance de l'imagination - débridée chez Jean Echenoz, vertigineuse chez Patrick Deville et dévorante chez Tanguy Viel. Le récit renouvelle ainsi sa puissance de fascination tout en exhibant les liens complexes entre la fiction et le réel et en permettant de « réfléchir à la question plus large du statut de l'imaginaire dans nos sociétés contemporaines ${ }^{25} »$.

\section{La comédie de l'illusion}

En affichant leur facticité, ces romans cherchent moins à dessiner les repères référentiels et les pôles axiologiques de la représentation du monde qu'à abolir les limites entre le vrai et le faux pour réfléchir sur les mécanismes de la représentation et de l'illusion; en cela, et selon les mots de Christine Baron, les romans « cessent de se demander ce qui constitue la fiction pour s'interroger sur ses usages et sur la signification anthropologique d'un désir de fiction dont la permanence historique est avérée ${ }^{26} \gg$. Ils exploitent ainsi leur spécularité pour nourrir une dynamique de l'improbable mais également pour faire du romanesque un objet conceptuel. Ce faisant, ils pointent du doigt les problématiques ontologiques posées par ce que Thomas Pavel appelle l'« axiologie du romanesque »: selon le

25. France Fortier et Andrée Mercier, op. cit, p. 181.

26. Christine Baron, «La notion de fiction », dans Jean Bessière (dir.), Littérature, représentation, fiction, Paris, Champion, 2007, p. 64. 
critique en effet, le romanesque est fondé sur « les valeurs qui se dessinent à l'horizon de notre vie morale ${ }^{27} \gg$, mais les trois œuvres proposent moins un réinvestissement de cette construction idéalisée qu'une interrogation de la puissance des normes et des représentations mentales sur l'existence. Elles explorent en effet les résonances de cette construction, non seulement pour en démystifier les faux-semblants, mais aussi pour poser la question du désir de fiction, de l'élaboration des représentations, du rôle des constructions mentales dans l'expérience humaine. Cette interrogation est particulièrement sensible dans trois romans de Patrick Deville, Le Feu d'artifice, La Femme parfaite et Ces deux-là, où le rapport aux stéréotypes finit par brouiller les repères entre la représentation et la réalité qu'elle désigne et engage une réflexion sur l'illusion pour souligner « l'extravagante complexité du réel », « l'artifice bigarré de la diversité du monde ${ }^{28}$ ». Plutôt qu'une condamnation univoque, la fiction prend ainsi en compte les illusions et les croyances qui constituent inévitablement la trame du monde, et entraîne le lecteur dans le vertige des apparences.

Comme le souligne Anne Chevalier, le roman contemporain choisit d'intégrer les stéréotypes romanesques plutôt que de les évacuer et sa valeur de recherche tient précisément « à ce qu'il fait apparaître [la stéréotypie] et en fait prendre conscience ${ }^{29} \gg$. La diégèse des trois romans est saturée de représentations factices du romanesque qui rendent sensible sous forme de discours figés tout un imaginaire idéaliste. Les clichés romanesques deviennent des rengaines obsédantes pour des personnages en perte de vitesse, et apparaissent régulièrement sous la forme de chansons fredonnées à voix basse («Si tu ne comprends pas Qu'il te faut revenir [...] Je ferai de nous deux Mes plus beaux souvenirs $\left.{ }^{30} »\right)$ ou de slogans répétés au gré de campagnes publicitaires ("No se puede vivir sin amar... », " La vie c'est l'amour... », "Love is what you need $\left.{ }^{31} . . . »\right)$. Ils sont présentés comme les résultats de tout un processus de construction, qu'ils soient créations musicales ou stratégies commerciales. Ces mises en abyme n'auront de cesse de montrer la place prépondérante des représentations mentales au sein de l'existence. Patrick Deville fait ainsi du manège des postures amoureuses le sujet de Ces deux-là et, interrogé sur son projet lors d'un entretien, il précise :

Il fallait que ce fût toujours la même vieille histoire éculée du tango, les couples se poursuivant tout autour de la piste comme autour de la

27. Thomas Pavel, «L'axiologie du romanesque », dans Gilles Declercq et Michel Murat (dir.), Le Romanesque, op. cit., p. 283-290.

28. Patrick Deville, Le Feu d'artifice, op. cit., p. 139.

29. Anne Chevalier, «La peur et le désir du romanesque », dans Alain Goulet (dir.), Le Stéréotype, Caen, Presses universitaires de Caen, p. 153-164.

30. Patrick Deville, Ces deux-là, op. cit., p. 14.

31. Ibid., p. 146. 
planète, adoptant les poses successives et codifiées de la pavane : l'avancée conquérante, menton dressé, le recul outragé, le dépit, choc du talon sur le plancher, l'abandon, la séparation, et toujours la chère vieille autodestruction $^{32}$.

De la femme fatale à l'aventurier idéaliste, les personnages se répartissent des rôles à l'axiologie transparente, dont la dimension mimétique est soulignée. L'imaginaire du cinéma vient par exemple illustrer ce réservoir de postures : dans Le Feu d'artifice, Juliette est assimilée à l'héroïne du film La Fille dangereuse de Guido Brignone, tandis que Louis, qui agit " comme il l'avait vu faire au cinéma ${ }^{33}$ ", tente de se fondre dans la peau d'un acteur hollywoodien et qu'Olga, l'héroïne de La Femme parfaite, est comparée à « une actrice d'âge mûr abandonnant un personnage d'ingénue au sortir de la scène ${ }^{34} \gg$. Les protagonistes vont jusqu'à s'inventer l'histoire de leur existence, en faisant de leurs chimères leur unique réalité, à l'image du narrateur du Feu d'artifice qui se rêve régisseur de sa propre vie pour en récrire le scénario, ou à l'image du narrateur de La Femme parfaite qui compile les signes - une photo, une poignée de cheveux, des produits de beauté - pour simuler une vie conjugale aux yeux de son entourage.

Face à la multiplication des représentations factices, le narrateur se dote d'une autorité herméneutique : sa lucidité face au leurre envahit la fiction pour dire l'effondrement des mythologies de la passion humaine et de l'action révolutionnaire, qui ne sont plus sensibles que sous forme de fragments discontinus ou de vestiges. La précarité de l'illusion est soulignée par un narrateur qui martèle sa volonté d'éviter le piège de l'idéalisme et qui met le romanesque à distance à travers un discours dépréciatif. $\mathrm{La}$ crédulité est incarnée par la figure insouciante et naïve de l'enfant - l'un court après un camion de cirque, l'autre détaille en criant les couleurs d'un feu d'artifice ou se rêve astronaute -, alors que le narrateur brisé par les échecs se fait le porte-parole d'une civilisation sur le déclin, celle «d'un pays riche et vieillissant ${ }^{35}$ ». Cet écart temporel entre le règne de l'illusion et l'empire de la clairvoyance prend acte d'un basculement à la fois intime - le passage à l'âge adulte - et collectif-l'échec des idéologies révolutionnaires - au sein d'une temporalité qui n'est plus appréhendée à travers la notion de progrès mais à travers celle de dégradation. Cet écart se double d'une mise à distance modale quand le narrateur stigmatise les illusions de « ces deux-là » qui « croient s'aimer encore et qui slaloment entre les flaques de boue ${ }^{36}$ » ou de cette autre qui, « sur un chemin de terre rouge dans une plaine à blé où coulait une rivière, les épis mûrs [dansant] sous le

32. Jean-Didier Wagneur, « Deville dans l'œuf du cyclone », op. cit.

33. Patrick Deville, Le Feu d'artifice, op. cit., p. 32.

34. Patrick Deville, La Femme parfaite, op. cit., p. 125.

35. Patrick Deville, Le Feu d'artifice, op. cit., p. 128.

36. Patrick Deville, Ces deux-là, op. cit., p. 11. 
vent d'orage, pensait que ce bonheur n'en finirait jamais ${ }^{37} \gg$. La conscience du leurre fait se déliter les motifs romanesques et le narrateur implique le lecteur dans ce processus de démystification par la multiplication des sousentendus visant à établir l'évidence du propos. Les appels à des connaissances ou des expériences partagées se multiplient et le narrateur ponctue son propos de marqueurs de complicité. «On connaît l'état de confusion mentale qui suit [la rencontre] ${ }^{38} »$, précise-t-il par exemple à propos de la naissance du sentiment amoureux, avant d'ajouter quelques pages plus loin : " On connaît les arguments fallacieux de la passion amoureuse ${ }^{39}$. » Ces procédés, qui fondent ce que Francis Langevin appelle un « discours de la connivence ${ }^{40} »$, établissent l'autorité d'un narrateur qui ancre sa crédibilité dans une complicité avec le lecteur pour se présenter comme maître des apparences.

La multiplication des discours rapportés à l'intérieur de la fiction répercute cette distanciation désabusée : les figures féminines des romans de Patrick Deville, Juliette, Antonia, Alfonsina ou encore Olga, stigmatisent comme « romanesques » des discours trompeurs dont elles ont fait leur deuil. Cependant, cette ligne de partage entre la fiction et la non-fiction se voit elle-même contredite par la multiplication des points de vue et par les revirements de l'illusion, car les différentes condamnations du romanesque se renvoient dos à dos. Si le romanesque, comme le souligne Alain Schaffner, est une catégorie spéculaire, qui repose sur une comparaison avec l'expérience de la réalité ${ }^{41}$, les fictions de Patrick Deville soulignent à quel point cette expérience constitue elle-même un repère instable, qui se révèle également assujetti à l'illusion. Alfonsina rejette ainsi la crédulité de ceux qui défendent l'authenticité du sentiment amoureux au nom d'une vision matérialiste de la passion:

Ah, les chansons d'amour, l'amour toujours, comme si elle allait y croire, elle qui passait sa vie à les écrire et à les chanter. Comme si elle allait tomber dans le panneau, elle qui était du côté des artistes, et de la vaste conspiration des artistes qui ont inventé de toutes pièces le mythe de l'amour. Qui bien sûr n'a jamais existé en dehors que dans les livres et dans les chansons ${ }^{42}$.

Mais le narrateur fait de cette lucidité proclamée une posture elle-même fallacieuse dont il se détache pour prôner l'hypothèse d'une manipulation non plus esthétique mais commerciale : aux chantres de l'amour, il oppose

37. Patrick Deville, La Femme parfaite, op. cit., p. 129.

38. Patrick Deville, Ces deux-là, op. cit., p. 82.

39. Ibid., p. 91.

40. Francis Langevin, «La connivence construite par le discours de l'évidence », Temps zéro, revue d'étude des écritures contemporaines, $\mathrm{n}^{\circ} 2$, [En ligne], http://tempszero.contemporain.info/document384 (consulté le 30 juin 2011).

41. Alain Schaffner, "Le romanesque : idéal du roman? », dans Michel Murat et Gilles Declercq (dir.), op. cit., p. 273.

42. Patrick Deville, Ces deux-là, op. cit., p. 94. 
la «théorie des focos », selon laquelle le sentiment amoureux ne serait qu'un instrument au service d'un « vaste réseau arachnéen destiné un jour à envelopper de son velours la lente révolution de la planète entière ${ }^{43}$ ". Ce fantasme hégémonique, incarné par l'agence matrimoniale mafieuse de la World Lovelies, se verra lui-même stigmatisé, dans une réversibilité infinie des expériences : «Elle n'y a jamais cru une seconde, bien sûr, à cette histoire de mafia, de bombes dans un bateau. Il avait dû taper ça le matin même sur son ordinateur, ivre d'amour pour elle. Elle avait trouvé ça très romanesque ${ }^{44}$. » L'intrigue articule sciemment plusieurs représentations subjectives de la réalité qui, parce qu'elles cherchent à imposer une géométrie au monde, sont forcément chimériques. Au sein de ce manège des représentations, le narrateur est trompé comme les autres, lui qui tombe même dans les pièges qu'il dénonce. Dans Le Feu d'artifice, il apparaît comme un personnage abusé par son propre discours et par le dispositif fictionnel qu'il met en place, voyant son héros devenir l'auteur d'une histoire où il est relégué au rang de personnage. De la même manière, le narrateur de La Femme parfaite est pris au piège de sa propre fiction, puisque l'invention d'une compagne le plongera au cœur d'une manipulation où il est accusé, à tort mais selon toute apparence, d'être l'instigateur d'un meurtre passionnel. La mise à distance du stéréotype romanesque perd de son sens face à cette réversibilité qui généralise la persistance des représentations mentales en dépit d'une volonté de détachement. Dès lors, l'adhésion à la fiction ne se situe plus au niveau de la consommation innocente du rêve, sans cesse empêchée, mais dans le vertige situé au cœur d'une accumulation de représentations toujours sur le point de se dissiper.

Chacun des protagonistes, le narrateur inclus, se réfère ainsi à une vision du monde qui ne coïncide pas avec celle des autres. L'attention portée aux chimères de chacun permet d'explorer les réalités intimes et imaginaires d'individus qui ne sont pas dupes de l'illusion, mais qui vivent en ayant connu le plaisir du rêve et savent qu'ils peuvent trouver dans la précarité d'un faux-semblant une sensation de plénitude. Dans Ces deux-là, les personnages se montrent ainsi prêts à succomber à nouveau au vieux mensonge de l'amour, pour l'avènement d'un instant de fulgurance où la naissance de la passion cö̈ncide avec la conscience de sa facticité, mais aussi avec le plaisir de sa gratuité. Le narrateur de Ces deux-là ne fait pas exception, lui qui suspend son savoir et souligne en voyant danser deux amants éconduits :

Ces deux-là tournoyaient maintenant comme les deux seuls vivants au milieu des ombres. Et sans doute les dieux multicolores et neurasthéniques de l'Asie n'avaient-ils combiné toute cette histoire que pour cette rencontrelà, de toute éternité les ouragans et les révolutions pour que ces deux-là,

43. Ibid., p. 128.

44. Ibid., p. 132. 
dans la lumière dorée d'une fin d'après-midi, dans un bungalow sur pilotis, à l'aplomb de la mer vermillonnée, dansent sur une arène minuscule devenue pour un instant le centre du monde ${ }^{45}$.

Le roman ne déploie pas des trajectoires qui verraient passer les personnages de l'aveuglement de la passion à une réalité providentielle, mais propose une errance circulaire qui n'empêche pas le surgissement de moments de suspension, souvent représentés par l'évocation du tournoiement, de l'extase ou du bouleversement, pendant lesquels les protagonistes ont l'impression d'être hors du temps. Si chacun reste fasciné par les représentations illusoires, c'est que celles-ci, quelle que soit leur nature, procurent la possibilité d'un bonheur à la fois « lamentable et merveilleux ${ }^{46}$ » et c'est pour cette raison que le narrateur de Ces deux-là, au seuil de la mort, se nourrit encore de romanesque en écoutant chanter « encore une fois, les yeux fermés, la survivante provisoire aux longs cheveux lisses et très noirs ${ }^{47} \gg$.

\section{« Le soupçon d'un petit fond irréductible de l'être "}

Si le romanesque est présent dans les trois œuvres étudiées ici, c'est avant tout parce que l'existence y est désignée comme une lutte contre l'emprise de modèles normatifs et de représentations mentales, mais aussi parce que celle-ci apparaît comme une invention intime qui prend nécessairement pour matériau les bribes de discours que les personnages croisent sur leur route, les images qui structurent leur intériorisation du monde. Le traitement des clichés, dans un mélange de drame et de drôlerie qui donne une place centrale au surgissement de l'imprévu, dit les enjeux passés et les résonances présentes d'une mythologie préconstruite tout en posant la question de ses influences à la fois destructrices et incontournables. En thématisant le rapport au romanesque, Patrick Deville prend en compte, pour dire cette « invention incroyable ${ }^{48}$ » qu'est l'existence, une aspiration à l'absolu qui persiste et se renouvelle en dépit de la conscience de sa fragilité. C'est peut-être aussi ce que tend à établir Jean Echenoz, chez qui le sentiment précaire de la présence au monde est paradoxalement lié à de rares moments d'abandon et pourrait être métaphorisé par l'image du couple suspendu, au bord d'une falaise dans Le Méridien de Greenwich, dans une cabine téléphérique dans Les Grandes Blondes. Cette précarité de l'existence se lit avec autant de force chez Tanguy Viel, où la volonté d' « être neuf », selon les termes du narrateur du Black Note, ne peut se réaliser sans passer par une réécriture romanesque assumée, comme le montre l'histoire romancée par laquelle le narrateur de Paris-Brest parvient à se libérer de l'influence familiale.

45. Ibid., p. 124.

46. Ibid., p. 126.

47. Ibid., p. 156-157.

48. Patrick Deville, La Femme parfaite, op. cit., p. 109. 
L'exploration thématique du romanesque apparaît dès lors moins comme une condamnation univoque des simulacres que comme une interrogation des rapports entre l'existence et l'imaginaire. La force de ces romans du romanesque, qui racontent la manière dont l'individu invente son histoire, est ainsi de générer une tension pragmatique entre distanciation et immersion, une oscillation ontologique entre imaginaire et réalité, un vertige axiologique entre destruction et salut : la voie possible entre ces hésitations n'est pas rejet de toute représentation mais réinvention du sens. À travers cette quête se donnent ainsi à lire à la fois la mise en doute des certitudes et la persistance, selon les mots de Patrick Deville, du « soupçon d'un petit fond irréductible de l'être ${ }^{49} »$, soupçon prenant ici valeur positive et devenant point d'ancrage d'une fiction esthétiquement et ontologiquement réhabilitée plutôt que source de dépouillement narratif.

Anne Sennhauser

Université Sorbonne nouvelle - Paris 3, Sorbonne Paris Cité, CERACC

49. Patrick Deville, «Entretien », dans Xavier Person et Henri Scepi, Patrick Deville, Poitiers, Office du tourisme de Poitou-Charentes, 1991, p. 28. 